\title{
Hydrocarbons in Industry
}

SINCE the structures and classical methods of synthesis of the aliphatic hydrocarbons serve as an admirable introduction to systematic organic chemistry, it is perhaps natural that these early chapters should be soon ignored and then forgotten. In those countries, however, fortunately endowed with natural sources of liquid hydrocarbons, their chemistry has become a subject of vital technical and national importance, whilst countries which have to rely on imported or synthetic products are even more dependent on methods by which cheapness of production and economy in utilization of fuels of high octane number or of lubricants can be achieved.

A general discussion was held by the Faraday Society on April 17-19 at the Imperial College of Science and Technology, London, under the title "Hydrocarbon Chemistry", with the object of ascertaining how far present scientific inquiry into the reactions of the hydrocarbons could serve in interpreting the somewhat complex processes already operating in industry. The discussion divided itself naturally into four sections : homogeneous thermal reactions, catalytic reactions, synthetic hydrocarbon production and, finally, olefine polymerization.

It is admitted that the mechanism of the thermal eracking process is a complicated one, but that it involves chain mechanisms at some stage, a hypothesis first advanced by Prof. R. O. Rice and now generally accepted. There is, however, no unanimity of opinion as to the actual chain processes involved in the reactions. Hydrocarbon free radicals can be prepared by a variety of methods and their properties have been described. It has also been shown that they can initiate chains of reaction when admitted to the saturated hydrocarbons at suitably elevated temperatures. Much of the discussion centred around the questions as to how the reaction centres originate, what were the relative rates of such reactions as

and

$$
\begin{aligned}
& \mathrm{H}+\mathrm{C}_{2} \mathrm{H}_{6} \rightarrow \mathrm{H}_{2}+\mathrm{C}_{2} \mathrm{H}_{5}, \\
& \mathrm{H}+\mathrm{C}_{2} \mathrm{H}_{6} \rightarrow \mathrm{CH}_{4}+\mathrm{CH}_{3},
\end{aligned}
$$

$$
\mathrm{H}_{2}+\mathrm{CH}_{3} \rightarrow \mathrm{H}+\mathrm{CH}_{4},
$$

and by what mechanisms admission of nitric oxide cuts down the velocity of decomposition.

From the technical point of view, the problems to be solved in connexion with the synthesis of fuels such as iso-octane or aromatic hydrocarbons are how to utilize two straight-chain hydrocarbon fractions, one from $\mathrm{C}_{6}-\mathrm{C}_{8}$ and the other from $\mathrm{C}_{1}-\mathrm{C}_{4}$. It appears clear that owing to the high temperatures involved in thermal cracking, which leads to loss of control, the method of attack by thermal decomposition of the $\mathrm{C}_{6}-\mathrm{C}_{9}$ fraction to $\mathrm{C}_{3}-\mathrm{C}_{4}$ and resynthesis of isoparaffins is not in favour, and alternative methods are being sought. Three avenues of attack on this problem are being explored, each involving catalytic reactions. The most direct involves catalytic cracking to a $\mathrm{C}_{3}-\mathrm{C}_{4}$ fraction followed by resynthesis of isoparaffins, the second catalytic ring closure, and the third attempts to bring about directly catalytic isomerism to isoparaffins as has already been found possible in the shorter members of the series. The discussion made it clear that the modus operandi of any one of these processes is by no means clear. This is exemplified by the fact that in one of the simplest reactions, namely, the exchange reaction of deuterium with an unsaturated hydrocarbon, such as ethylene, or a saturated one, such as ethane, three different mechanisms have been advanced. These may be depicted as follows :

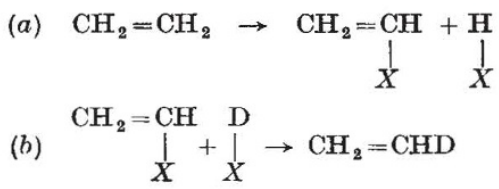

(2)

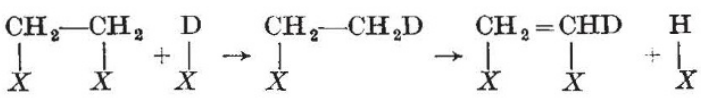

(3)

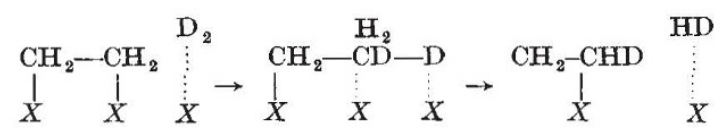

A similar diversity of opinion was noted when the mechanism of ring closure was discussed.

When heptane is passed over a dehydrogenating catalyst either of the oxide or metal type to produce toluene, a mono-olefine is found to be present. The third mechanism described above for the exchange reaction is likewise applicable to the process of olefine formation, but we note that the half hydrogenated state is an intermediary in the reaction. The answer to the question whether the cyclization proceeds through the olefine or through the half hydrogenated state awaits further experiment. It is also interesting to observe that both (2) and (3) provide us with mechanisms by which the double bond in an olefine can travel towards the end of the chain, so 
that cyclization may result. Cyclization in the gas phase involves a large change in entropy, since the difference in the internal mobilities of the straight chain and ring is so pronounced; it is clearly important to devise some method by which the position of the molecule adsorbed on the catalytic surface at the temperature of ring closure may be examined.

On the synthesis side, discussion was devoted to the interesting reaction which occurs between a saturated tertiary hydrocarbon and an olefine in the presence of a variety of catalytic agents, especially sulphuric acid. Whilst considerable progress has been made in elucidating the structure and energies of reactions involving conjugated double bonds, it would be very desirable if calculations on reactions of the type

$$
\begin{aligned}
& \mathrm{D}+\mathrm{CH}_{*} \rightarrow \mathrm{CH}_{3} \mathrm{D}+\mathrm{H}, \\
& \mathrm{D}+\mathrm{CH}_{4} \rightarrow \mathrm{CH}_{3}+\mathrm{HD},
\end{aligned}
$$

were made. In the technical synthesis of liquid fuel, it is clear that processes of coal hydrogenation are extremely complex and that the identification and examination of the various reactions involved would be a matter of great difficulty. Whilst very complex products can be made by the Fischer-Tropsch process, the initial reactions which the carbon monoxide and hydrogen undergo in building up the complex hydrocarbons are much simpler, and some success has already been achieved in identifying and examining the successive reactions which are involved. It was clear from the discussion that it may be possible to synthesize many other products in addition to the straight-chain hydrocarbons by means of this elegant reaction.

The last section of the discussion was devoted to the polymerization of the olefines. Here dimerization of short-chain olefines to produce fuels, and polymerization to produce long-chain macromolecules suitable for plastics, are at present the two important technical objectives. No less than nine papers were devoted to this aspect of hydrocarbon chemistry.

The Faraday Society always attempts to emphasize the international character of its discussions on scientific subjects, and it is worthy of comment that this meeting was a truly representative one in spite of the present political situation.

Eric K. Rideal.

\section{Growth of Induced Plant Tumours}

\section{By László J. Havas, Institute of Pathological Anatomy, Brussels}

\begin{abstract}
A COMPARISON between the malignant A tumours of animals and the plant tumours, commonly called 'crown gall', is beyond the scope of the present article*. It may be pointed out, however, that characteristic histological and cytological differences can be observed-obviously enough-between these two pathological structures, such differences being inherent in the two types of host organisms themselves. It may be added further that the analogies of response of plants and animals, to be described, were obtained in spite of their structural differences.

Inspired, no doubt, by the experimental induction of tar tumours in animals, discovered by Yamagiva and Ichikawa, Komuro ${ }^{1}$ exposed the root tips of Vicia Faba and Pisum sativum to suspensions of coal tar. He obtained by this method well-characterized neoplasic structures termed by him 'phyto-tartumours'. Most of the cellular abnormalities described by Komuro in these swellings, such as giant and multinucleate cells, plurinucleolated, lobed, vacuolized and fragmented

\footnotetext{
* See for reference on this subject the excellent review of A. J.
} Riker and T. O. Berge, Amer. J. Cancer, 25, No. 2, 310-356.
\end{abstract}

nuclei, amitotic divisions, chromosome aberrations, etc., were also observed by Mottram, Picon, Levine and Bergmann ${ }^{2}$ and others, in onion and bean roots treated with coal tar and also with Scharlach Red. Such effects, as the systematic investigations of Levine ${ }^{3,4}$ and Chargaff ${ }^{5}$ have shown, were not limited to the particularly sensitive root tips, for swellings macroscopically comparable to crown gall, and presenting similar cytological abnormalities, were also produced by them on the stems of the most heterogeneous annuals, and even woody plants, subjected to local treatments (painting) with the above and a variety of other chemicals.

The discovery of the active factors in coal tar responsible for tumour production in animals (Bloch and Widmer), and the isolation and synthesis of these and other highly carcinogenic hydrocarbons (Kennaway; Clar; Cook et al. ${ }^{6}$, Fieser, and others), gave an added impetus to the study of the etiology and of the experimental induction of chemical cancers in animals. One of these compounds, $1: 2: 5: 6:$ dibenzanthracene, was tested by Levine ${ }^{3}$, and was shown 